\title{
Modern Encryption and Decryption Algorithm based on ASCII Value and Binary Operations
}

\author{
Deepak \\ Bahra University \\ Shimla Hills, Himachal Pradesh \\ India
}

\begin{abstract}
Security is the most important issue from last few decades. Cryptography plays an important role in security. Encryption and Decryption came as a solution against security threats. There are so many proposed algorithms by researchers like AES,DES,RSA and many more but they are lacking somewhere in terms of security. Therefore cryptography demands such encryption and decryption algorithms which are real hard to crack. In this paper, an algorithm has been proposed based on ASCII values and binary operations for both encryption and decryption to enhance the security so that the attackers might not easily crack the logic to reach the original message sent via network.
\end{abstract}

\section{General Terms}

Cryptography, Encryption, Decryption, Algorithm etc.

\section{Keywords}

ASCII, Binary, Encryption, Decryption, Symmetric Encryption Algorithm, Plain Text, Cipher Text

\section{INTRODUCTION}

The word "cryptography" is made of two Greek roots: kryptos, meaning secret, and graphos, meaning writing. Originally, "secret writing" was the main topic for those who studied in this area. However, secret writing is no longer the only focus to cryptographic research. In fact, our work has gone far beyond that. ${ }^{[1]}$ Cryptography played a major role in the course of World War II, and some of the first working computers were dedicated to cryptanalytic tasks. ${ }^{[2]}$ Cryptography has recently played a significant role in secure data transmissions and storages. Most conventional data encryption schemes are relatively complicated and complexity in encrypted keys is insufficient, resulting in long computational time and low degree of security against all types of attacks. Consequently, a highly secured and robust data encryption scheme is necessary. ${ }^{[8]}$

Encryption is basically a process or algorithm to make information hidden or secret. It is considered as the subset of cryptography. It is the actual process of applying cryptography. It is the process to transform or converting the data into some another form that appears to be random, meaningless and unintelligible. It can also be said that encryption is the process of transforming plaintext into the cipher text where plaintext is the input to the encryption process and cipher text is the output of the encryption process. ${ }^{[5]}$ Decryption is the reverse process of Encryption. In decryption, at receiver side, cipher text is converted to plaintext by using secret key and performing operations.

In computer systems, the algorithm consists of complex mathematical formulas that dictate the rules of conversion process from plain text to cipher text and vice versa combined

\author{
Parveen \\ Bahra University \\ Shimla Hills, Himachal Pradesh \\ India
}

with the key. However, some of the encryption and decryption algorithms use the same key (i.e. sender and receiver). ${ }^{[6]}$

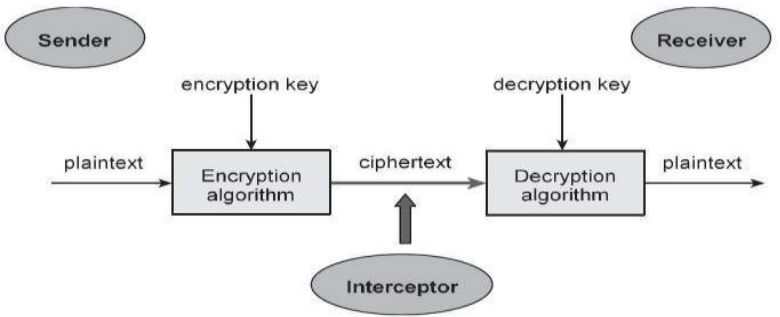

Fig 1: Model of Cryptosystem

\section{ENCRYPTION ALGORITHM}

This algorithm is used to encrypt data by using ASCII values of the data to be encrypted. The secret key used is same for both encryption and decryption of the message. So this is a symmetric encryption algorithm. Length of the secret key need not to be the same as the length of plain text but it can be made equal.

\section{Assumptions:}

$\mathrm{p}[\mathrm{i}]$ be an array that stores binary equivalent of plain text of ith position.

$s[j]$ be an array that stores binary equivalent of secret key of jth position.

$\mathrm{e}[\mathrm{k}]$ be an array that stores binary equivalent of results after operation of kth position.

Plain Text: LIFE IS A JOURNEY NOT A RACE.

Secret Key: SUCCESS

Step 1: Give input as plain text and store plain text in the form of String.

\section{Plain Text: LIFE IS A JOURNEY NOT A RACE}

Step 2: Apply permutation on the string and place the words at odd position first and words at even position after placing odd positioned words. Now plain text will become (Assuming first word at zeroth position) :

\section{Plain Text: IS JOURNEY A LIFE A NOT RACE}

Step 3: Remove white spaces from the new plain text i.e. spaces and use the given secret key for each character. If secret key is short then apply repetitions in the secret key so that each character in plain text get a character from secret key.

Plain Text: ISJOURNEYALIFEANOTRACE

\section{KEY: SUCCESSSUCCESSSUCCESSS}

Step 4: Find ASCII value of each character for both Plain text and Key. Convert ASCII values into its binary equivalent (8 bits). 
Table 1. ASCII Value and its Binary Equivalent used in Text

\begin{tabular}{|c|c|c|}
\hline Character & ASCII Value & Binary Equivalent \\
\hline I & 73 & 01001001 \\
\hline S & 83 & 01010011 \\
\hline J & 74 & 01001010 \\
\hline O & 79 & 01001111 \\
\hline U & 85 & 01010101 \\
\hline R & 82 & 01010010 \\
\hline N & 78 & 01001110 \\
\hline E & 69 & 01000101 \\
\hline Y & 89 & 01011001 \\
\hline A & 65 & 01000001 \\
\hline L & 76 & 01001100 \\
\hline F & 70 & 01000110 \\
\hline T & 84 & 01010100 \\
\hline C & 67 & 01000011 \\
\hline
\end{tabular}

Step 5: Perform addition on ASCII binary equivalent for each character of plain text and secret key which are at similar positions.

Table 2. Table Depicting Addition Operation

\begin{tabular}{|c|c|c|c|}
\hline Position & $\begin{array}{c}\text { Binary } \\
\text { Equivalent of } \\
\text { Plain Text at } \\
\text { Given } \\
\text { Position i.e. } \\
\text { p[i] }\end{array}$ & $\begin{array}{c}\text { Binary } \\
\text { Equivalent of } \\
\text { Secret Key at } \\
\text { Given } \\
\text { Position i.e. } \\
\text { s[j] }\end{array}$ & $\begin{array}{c}\text { Addition } \\
\text { of Both } \\
\text { Binary } \\
\text { Equivalen } \\
\text { ts i.e. e[k] }\end{array}$ \\
\hline 0 & 01001001 & 01010011 & 10011100 \\
\hline 1 & 01010011 & 01010101 & 10101000 \\
\hline 2 & 01001010 & 01000011 & 10001101 \\
\hline 3 & 01001111 & 01000011 & 10010010 \\
\hline 4 & 01010101 & 01000101 & 10011010 \\
\hline 5 & 01010010 & 01010011 & 10100101 \\
\hline 6 & 01001110 & 01010011 & 10100001 \\
\hline 7 & 01000101 & 01010011 & 10011000 \\
\hline 8 & 01011001 & 01010101 & 10101110 \\
\hline 9 & 01000001 & 01000011 & 10000100 \\
\hline 10 & 01001100 & 01000011 & 10001111 \\
\hline 11 & 01001001 & 01000101 & 10001110 \\
\hline 12 & 01000110 & 01010011 & 10011001 \\
\hline 13 & 01000101 & 01010011 & 10011000 \\
\hline 14 & 01000001 & 01010011 & 10010100 \\
\hline 15 & 01001110 & 01010101 & 10100011 \\
\hline 16 & 01001111 & 01000011 & 10010010 \\
\hline 17 & 01010100 & 01000011 & 10010111 \\
\hline 18 & 01010010 & 01000101 & 10010111 \\
\hline 19 & 01000001 & 01010011 & 10010100 \\
\hline 20 & 01000011 & 01010011 & 10010110 \\
\hline 21 & 01000101 & 01010011 & 10011000 \\
\hline
\end{tabular}

Step 6: Now find the 2's complement of e[k] (Use Step 5 Table for $\mathrm{e}[\mathrm{k}])$
Table 3. 2's complement Table for Binary Equivalent

\begin{tabular}{|c|c|c|}
\hline Position & $\begin{array}{c}\text { Addition of Both } \\
\text { Binary Equivalents } \\
\text { i.e. } \mathrm{e}[\mathrm{k}]\end{array}$ & $\begin{array}{c}\text { 2's complement of } \\
\text { e[k] }\end{array}$ \\
\hline 0 & 10011100 & 01100010 \\
\hline 1 & 10101000 & 01010110 \\
\hline 2 & 10001101 & 01110001 \\
\hline 3 & 10010010 & 01101100 \\
\hline 4 & 10011010 & 01100100 \\
\hline 5 & 10100101 & 01011001 \\
\hline 6 & 10100001 & 01011101 \\
\hline 7 & 10011000 & 01100110 \\
\hline 8 & 10101110 & 01010000 \\
\hline 9 & 10000100 & 01111010 \\
\hline 10 & 10001111 & 01101111 \\
\hline 11 & 10001110 & 01110000 \\
\hline 12 & 10011001 & 01100101 \\
\hline 13 & 10011000 & 01100110 \\
\hline 14 & 10010100 & 01101010 \\
\hline 15 & 10100011 & 01011011 \\
\hline 16 & 10010010 & 01101100 \\
\hline 17 & 10010111 & 01100111 \\
\hline 18 & 10010111 & 01100111 \\
\hline 19 & 10010100 & 01101010 \\
\hline 20 & 10010110 & 01101000 \\
\hline 21 & 10011000 & 01100110 \\
\hline
\end{tabular}

Step 7: The encrypted text corresponding to plain text in bit format will become (say new e[k])

01100010010101100111000101101100011001000101100101 01110101100110010100000111101001101111011100000110 01010110011001101010010110110110110001100111011001 11011010100110100001100110

Now apply 2 bit circular right shift

10001001010110011100010110110001100100010110010101 11010110011001010000011110100110111101110000011001 01011001100110101001011011011011000110011101100111 01101010011010000110011001

Step 8: Now pair 8 bits for each index position in same order and convert the bits into corresponding decimal number. Assume this decimal number as ASCII value and convert ASCII value to respective character or symbol.

Table 4. Conversion to ASCII Value and Corresponding Character

\begin{tabular}{|c|c|c|c|}
\hline Position & $\begin{array}{c}\text { Circular } \\
\text { Right } \\
\text { Shift of } \\
\text { e[k] }\end{array}$ & $\begin{array}{c}\text { ASCII } \\
\text { Value for } \\
\text { e[k] }\end{array}$ & $\begin{array}{c}\text { Corresponding } \\
\text { Character for } \\
\text { ASCII value }\end{array}$ \\
\hline 0 & 10001001 & 137 & ë \\
\hline 1 & 01011001 & 89 & Y \\
\hline 2 & 11000101 & 197 & † \\
\hline 3 & 10110001 & 177 & " \\
\hline
\end{tabular}




\begin{tabular}{|c|c|c|c|}
\hline 4 & 10010001 & 145 & $\mathfrak{x}$ \\
\hline 5 & 01100101 & 101 & $\mathrm{e}$ \\
\hline 6 & 01110101 & 117 & $\mathrm{u}$ \\
\hline 7 & 10011001 & 153 & $\ddot{O}$ \\
\hline 8 & 01000001 & 65 & A \\
\hline 9 & 11101001 & 233 & $\bar{U}$ \\
\hline 10 & 10111101 & 189 & $\phi$ (cent symbol) \\
\hline 11 & 11000001 & 193 & $\perp$ \\
\hline 12 & 10010101 & 149 & ò \\
\hline 13 & 10011001 & 153 & $\ddot{O}$ \\
\hline 14 & 10101001 & 169 & $\begin{array}{l}\text { () (Registered } \\
\text { trademark } \\
\text { symbol) }\end{array}$ \\
\hline 15 & 01101101 & 109 & $\mathrm{~m}$ \\
\hline 16 & 10110001 & 177 & 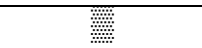 \\
\hline 17 & 10011101 & 157 & $\varnothing$ \\
\hline 18 & 10011101 & 157 & $\varnothing$ \\
\hline 19 & 10101001 & 169 & $\begin{array}{c}\text { ( ) (Registered } \\
\text { trademark } \\
\text { symbol) }\end{array}$ \\
\hline 20 & 10100001 & 161 & 1 \\
\hline 21 & 10011001 & 153 & $\ddot{O}$ \\
\hline
\end{tabular}

The text after encryption will be

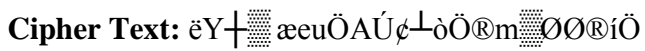

\section{DECRYPTION ALGORITHM}

Assumptions:-

$1[\mathrm{k}]$ be an array that stores updated binary equivalent of ciphertext of kth position.

$\mathrm{a}[\mathrm{j}]$ be an array that stores binary equivalent of secret key of jth position.

$\mathrm{b}[\mathrm{i}]$ be an array that stores subtraction of $\mathrm{l}[\mathrm{k}]$ and $\mathrm{a}[\mathrm{j}]$ of ith position.

Cipher Text: ëY+

Secret Key: SUCCESS

Step 1: Assign each character a position starting from 0 . Convert the each character of cipher text into the ASCII value and convert that ASCII value into binary

Table 4. Conversion to Characters to Binary Equivalent

\begin{tabular}{|c|c|c|c|}
\hline Position & $\begin{array}{c}\text { Character of } \\
\text { Cipher Text } \\
\text { at Given } \\
\text { Position }\end{array}$ & $\begin{array}{c}\text { ASCII } \\
\text { Value }\end{array}$ & $\begin{array}{c}\text { Binary } \\
\text { Equivalent }\end{array}$ \\
\hline 0 & $\ddot{\mathrm{e}}$ & 137 & 10001001 \\
\hline 1 & $\mathrm{Y}$ & 89 & 01011001 \\
\hline 2 & + & 197 & 11000101 \\
\hline 3 & $\mathrm{x}$ & 177 & 10110001 \\
\hline 4 & $\mathrm{e}$ & 145 & 10010001 \\
\hline 5 & $\mathrm{u}$ & 117 & 01100101 \\
\hline 6 & $\ddot{O}$ & 153 & 10011001 \\
\hline 7 & $\mathrm{~A}$ & 65 & 01000001 \\
\hline 8 & & &
\end{tabular}

\begin{tabular}{|c|c|c|c|}
\hline 9 & Ú & 233 & 11101001 \\
\hline 10 & $\phi$ (cent symbol) & 189 & 10111101 \\
\hline 11 & $\perp$ & 193 & 11000001 \\
\hline 12 & ò & 149 & 10010101 \\
\hline 13 & Ö & 153 & 10011001 \\
\hline 14 & $\begin{array}{c}\text { ( }) \text { (Registered } \\
\text { trademark } \\
\text { symbol) }\end{array}$ & 169 & 10101001 \\
\hline 15 & $\mathrm{~m}$ & 109 & 01101101 \\
\hline 16 & (1) & 177 & 10110001 \\
\hline 17 & $\varnothing$ & 157 & 10011101 \\
\hline 18 & $\varnothing$ & 157 & 10011101 \\
\hline 19 & $\begin{array}{c}\text { ( }) \text { (Registered } \\
\text { trademark } \\
\text { symbol) }\end{array}$ & 169 & 10101001 \\
\hline 20 & í & 161 & 10100001 \\
\hline 21 & Ö & 153 & 10011001 \\
\hline
\end{tabular}

Step 2: Apply 2 bit left circular shift on binary equivalent

\section{Binary Equivalent}

10001001010110011100010110110001100100010110010101 11010110011001010000011110100110111101110000011001 01011001100110101001011011011011000110011101100111 01101010011010000110011001

After applying 2 bit left circular shift

01100010010101100111000101101100011001000101100101 01110101100110010100000111101001101111011100000110 01010110011001101010010110110110110001100111011001 11011010100110100001100110

Step 3: Break the binary text into pairs of 8 bit for each position and apply 2's complement on each pair

Table 5. 2's Complement of Binary Equivalent

\begin{tabular}{|c|c|c|}
\hline Position & $\begin{array}{l}\text { Circular Left } \\
\text { Shift of } 1[k]\end{array}$ & $\begin{array}{c}\text { 2's complement } \\
\text { of } I[k]\end{array}$ \\
\hline 0 & 01100010 & 10011100 \\
\hline 1 & 01010110 & 10101000 \\
\hline 2 & 01110001 & 10001101 \\
\hline 3 & 01101100 & 10010010 \\
\hline 4 & 01100100 & 10011010 \\
\hline 5 & 01011001 & 10100101 \\
\hline 6 & 01011101 & 10100001 \\
\hline 7 & 01100110 & 10011000 \\
\hline 8 & 01010000 & 10101110 \\
\hline 9 & 01111010 & 10000100 \\
\hline 10 & 01101111 & 10001111 \\
\hline 11 & 01110000 & 10001110 \\
\hline 12 & 01100101 & 10011001 \\
\hline 13 & 01100110 & 10011000 \\
\hline 14 & 01101010 & 10010100 \\
\hline 15 & 01011011 & 10100011 \\
\hline 16 & 01101100 & 10010010 \\
\hline 17 & 01100111 & 10010111 \\
\hline
\end{tabular}




\begin{tabular}{|l|l|l|}
\hline 18 & 01100111 & 10010111 \\
\hline 19 & 01101010 & 10010100 \\
\hline 20 & 01101000 & 10010110 \\
\hline 21 & 01100110 & 10011000 \\
\hline
\end{tabular}

Step 4: Use the Secret key so that each text will get a character from Secret Key if number of character in Secret Key is less than the number of character in Cipher Text then apply repetition in secret key. Convert each character of secret key into ASCII value and then its binary equivalent.

\section{Key: SUCCESSSUCCESSSUCCESSS}

Table 6. Table depicting Updated Cipher Text Equivalent and Binary Equivalent of Secret Key

\begin{tabular}{|c|c|c|}
\hline Position & $\begin{array}{c}\text { Updated } \\
\text { Cipher Text } \\
\text { I[k] }\end{array}$ & $\begin{array}{l}\text { Binary Equivalent of } \\
\text { Secret Key at Given } \\
\text { Position i.e. a[j] }\end{array}$ \\
\hline 0 & 10011100 & 01010011 \\
\hline 1 & 10101000 & 01010101 \\
\hline 2 & 10001101 & 01000011 \\
\hline 3 & 10010010 & 01000011 \\
\hline 4 & 10011010 & 01000101 \\
\hline 5 & 10100101 & 01010011 \\
\hline 6 & 10100001 & 01010011 \\
\hline 7 & 10011000 & 01010011 \\
\hline 8 & 10101110 & 01010101 \\
\hline 9 & 10000100 & 01000011 \\
\hline 10 & 10001111 & 01000011 \\
\hline 11 & 10001110 & 01000101 \\
\hline 12 & 10011001 & 01010011 \\
\hline 13 & 10011000 & 01010011 \\
\hline 14 & 10010100 & 01010011 \\
\hline 15 & 10100011 & 01010101 \\
\hline 16 & 10010010 & 01000011 \\
\hline 17 & 10010111 & 01000011 \\
\hline 18 & 10010111 & 01000101 \\
\hline 19 & 10010100 & 01010011 \\
\hline 20 & 10010110 & 01010011 \\
\hline 21 & 10011000 & 01010011 \\
\hline
\end{tabular}

Step 5: Subtract binary equivalent of Secret key from Updated Cipher text for same positions

Table 7. Subtraction Operation between $1[k]$ and $a[j]$

\begin{tabular}{|c|c|c|c|}
\hline Position & $\begin{array}{c}\text { Updated } \\
\text { Cipher } \\
\text { Text I[k] }\end{array}$ & $\begin{array}{c}\text { Binary } \\
\text { Equivalent of } \\
\text { Secret Key at } \\
\text { Given Position } \\
\text { i.e. a[j] }\end{array}$ & $\begin{array}{c}\text { Binary } \\
\text { Equivalent of } \\
\text { Subtraction } \\
\text { operation at } \\
\text { Given Position i.e. } \\
\text { b[i] }\end{array}$ \\
\hline 0 & 10011100 & 01010011 & 01001001 \\
\hline 1 & 10101000 & 01010101 & 01010011 \\
\hline 2 & 10001101 & 01000011 & 01001010 \\
\hline
\end{tabular}

\begin{tabular}{|l|l|l|l|}
\hline 3 & 10010010 & 01000011 & 01001111 \\
\hline 4 & 10011010 & 01000101 & 01010101 \\
\hline 5 & 10100101 & 01010011 & 01010010 \\
\hline 6 & 10100001 & 01010011 & 01001110 \\
\hline 7 & 10011000 & 01010011 & 01000101 \\
\hline 8 & 10101110 & 01010101 & 01011001 \\
\hline 9 & 10000100 & 01000011 & 01000001 \\
\hline 10 & 10001111 & 01000011 & 01001100 \\
\hline 11 & 10001110 & 01000101 & 01001001 \\
\hline 12 & 10011001 & 01010011 & 01000110 \\
\hline 13 & 10011000 & 01010011 & 01000101 \\
\hline 14 & 10010100 & 01010011 & 01000001 \\
\hline 15 & 10100011 & 01010101 & 01001110 \\
\hline 16 & 10010010 & 01000011 & 01001111 \\
\hline 17 & 10010111 & 01000011 & 01010100 \\
\hline 18 & 10010111 & 01000101 & 01010010 \\
\hline 19 & 10010100 & 01010011 & 01000001 \\
\hline 20 & 10010110 & 01010011 & 01000011 \\
\hline 21 & 10011000 & 01010011 & 01000101 \\
\hline & & & \\
\hline
\end{tabular}

Step 6: Convert the resultant binary equivalent obtained after subtraction into ASCII values and corresponding character

Table 8. Conversion of ASCII Value to Character

\begin{tabular}{|c|c|c|c|}
\hline Position & $\begin{array}{c}\text { Resultant } \\
\text { Binary } \\
\text { Equivalent } \\
\text { i.e. b[i] } \\
\end{array}$ & $\begin{array}{c}\text { ASCII value } \\
\text { of Binary } \\
\text { Equivalent }\end{array}$ & $\begin{array}{c}\text { Correspond } \\
\text { ing } \\
\text { Character }\end{array}$ \\
\hline 0 & 01001001 & 73 & $\mathrm{I}$ \\
\hline 1 & 01010011 & 83 & $\mathrm{~S}$ \\
\hline 2 & 01001010 & 74 & $J$ \\
\hline 3 & 01001111 & 79 & $\mathrm{O}$ \\
\hline 4 & 01010101 & 85 & $\mathrm{U}$ \\
\hline 5 & 01010010 & 82 & $\mathrm{R}$ \\
\hline 6 & 01001110 & 78 & $\mathrm{~N}$ \\
\hline 7 & 01000101 & 69 & $\mathrm{E}$ \\
\hline 8 & 01011001 & 89 & $\mathrm{Y}$ \\
\hline 9 & 01000001 & 65 & A \\
\hline 10 & 01001100 & 76 & $\mathrm{~L}$ \\
\hline 11 & 01001001 & 73 & I \\
\hline 12 & 01000110 & 70 & $\mathrm{~F}$ \\
\hline 13 & 01000101 & 69 & $\mathrm{E}$ \\
\hline 14 & 01000001 & 65 & A \\
\hline 15 & 01001110 & 78 & $\mathrm{~N}$ \\
\hline 16 & 01001111 & 79 & $\mathrm{O}$ \\
\hline 17 & 01010100 & 84 & $\mathrm{~T}$ \\
\hline 18 & 01010010 & 82 & $\mathrm{R}$ \\
\hline 19 & 01000001 & 65 & A \\
\hline 20 & 01000011 & 67 & $\mathrm{C}$ \\
\hline 21 & 01000101 & 69 & $\mathrm{E}$ \\
\hline
\end{tabular}




\section{Text : ISJOURNEYALIFEANOTRACE}

Step 7: Use white spaces between the text wherever possible to form a meaningful word.

\section{Text : IS JOURNEY A LIFE A NOT RACE}

Step 8: Count the number of words in the text. First half of the text are those words which are at odd positions(in same order) and second half contains those words which are at even positions in the text (in same order). Apply permutation to rearrange them at the respective position.

Text : LIFE IS A JOURNEY NOT A RACE.

Thus plain text obtained as output is:

Plain text : LIFE IS A JOURNEY NOT A RACE.

\section{ADVANTAGE OF ASCII VALUE BASED ENCRYPTION AND DECRYPTION ALGORITHM}

In this approach, we can have same encrypted characters for different characters of the plain text. In the above example "LIFE IS A JOURNEY NOT A RACE", T and R from words NOT and RACE respectively and have same encrypted character which is $\varnothing$. So, if anyone trying to decrypt the text he would definitely face the problem as there can be many possibilities (meaning) for similar character decryption. There may be same encryption for similar cipher character but if the encryption is not same then an unauthorized user will have to apply so many permutations which will consume a lot of time to decode that message.

\section{LIMITATION}

There is a possibility that there might occur a character during encryption in Step 8 for any position of Encryption algorithm for which corresponding ASCII value and character does not exist. In that case, we can use any special character (like !,@,\#,\$,\% etc. which are rarely used) to assign a ASCII character at that position.

\section{CONCLUSION AND FUTURE WORK}

Everyone needs to protect himself in today's World as this is the era of digitalization. .People are lacking somewhere in terms of security of the data. Latest example of security threat was "WannaCry ransomware attack", which targeted computers running the Microsoft Windows Operating system by encrypting data and demanding ransom payments in the
Bitcoin cryptocurrency. So, goal should be to encrypt a message in such a way that it cannot be decrypted by anyone. Therefore, this ASCII value based encryption and decryption algorithm is a good alternative for data security. This algorithm shall be really helpful in applications like Cryptography, Network Security (Wired and Wireless), Encrypting and Decrypting etc.

The work proposed here can be further enhanced to make the algorithm more secure and the researchers are working in the field of Cryptography and developing many more algorithms.

\section{REFERENCES}

[1] New Tools in Cryptography: Mutually Indepent Commitments, Tweakable Block Ciphers, and Plaintext Awareness via Key Registration by Moses Liskov.

[2] Cryptography and Machine Learning Ronald L. Rivest* Laboratory for Computer Science Massachusetts Institute of Technology Cambridge, MA 02139

[3] The RC5 Encryption Algorithm Ronald L Rivest MIT Laboratory for Computer Science Technology Square, Cambridge, Mass. 02139.

[4] D. E. Denning. Cryptography and Data Security. Addison-Wesley, Reading, Mass., 1982.

[5] A Research paper: An ASCII value based data encryption algorithm and its comparison with other symmetric data encryption algorithms.

[6] Obaida Mohammad Awad Al-Hazaimeh "A NEW APPROACH FOR COMPLEX ENCRYPTING AND DECRYPTING DATA" International Journal of Computer Networks \& Communications (IJCNC) Vol.5, No.2, March 2013

[7] Dr. Prerna Mahajan \& Abhishek Sachdeva IITM, India "A Study of Encryption Algorithms AES, DES and RSA for Security" Global Journal of Computer Science and Technology Network, Web \& Security Volume 13 Issue 15 Version 1.0 Year 2013

[8] Warakorn Srichavengsup and Wimol San-Um "Data Encryption Scheme Based on Rules of Cellular Automata and Chaotic Map Function for Information Security" International Journal of Network Security, Vol.18, No.6, PP.1130-1142, Nov. 2016

[9] 